\title{
Inflammatory joint disease: a comparison of liposome scanning, bone scanning, and radiography
}

\author{
M M O'SUllivaN, ${ }^{1} \mathrm{~N}$ POWELL, ${ }^{2}$ A P FRENCH, ${ }^{3} \mathrm{~K}$ E WILliamS . \\ J R MORGAN ${ }^{+}$AND B D WILLIAMS
}

From the Departments of ${ }^{1}$ Rheumatology, ${ }^{2}$ Radiology, ${ }^{3}$ Medical Physics, and ${ }^{4}$ Medical Microbiology, University Hospital of Wales, Cardiff

SUMMARY Patients with rheumatoid arthritis, psoriatic arthritis, and osteoarthritis were assessed by clinical evaluation, radiography, and joint scintigraphy using technetium labelled methylene diphosphonate (MDP) and technetium labelled liposomes. Although both scanning techniques were more sensitive than radiographs in detecting joint disease, the liposomes scans were positive only in clinically active inflammatory disease. In patients with rheumatoid arthritis liposome scintigraphy was also able to discriminate between different grades of joint tenderness. In inactive inflammatory polyarthropathies, although the MDP bone scans continued to show increased activity, the liposome scans did not and were therefore a more accurate reflection of the clinical state. The increased uptake in the liposome scans may be due to incorporation of the liposomes into the phagocytic cells of the synovium. This scan may, therefore, by reflecting the activity of cells involved in the disease process, provide a useful way of assessing disease activity and progression.

Key words: rheumatoid arthritis, psoriatic arthritis, osteoarthritis, radionuclide scintigraphy, liposomes.

Liposomes are microscopic vesicles containing aqueous compartments separated by phospholipid bilayers. ${ }^{1}$ These particles are taken up by the phagocytic cells of the reticuloendothelial system, and their uptake can be demonstrated in vivo using liposomes radiolabelled with gamma emitting radionuclides such as technetium (Tc). ${ }^{2}$ Tc labelled liposomes have been used in attempts to identify macrophages surrounding tumour tissue ${ }^{3}$ and deep seated infection. ${ }^{+}$More recently we have shown that radiolabelled liposomes can be used to identify the inflammatory tissue associated with rheumatoid arthritis. ${ }^{5}$ Gammacamera joint scintigraphy with bone seeking radioactive isotopes has also been used to identify early inflammatory changes in a variety of arthropathies and shows abnormalities before the appearance of radiographic changes. ${ }^{67}$ The precise mechanisms which lead to positive scintigraphs in the liposome and bone scans are unclear, and in our previous studies we suggested that the mechanisms are different. ${ }^{8}$

Accepted for publication 31 October 1987.

Correspondence to Dr M M O'Sullivan. Rheumatology Department. University Hospital of Wales. Cardiff CF4 4XW.
In this paper we extend our preliminary observations on patients with rheumatoid arthritis to include other inflammatory polyarthropathies, and we compare the appearance obtained using technetium radiolabelled methylene diphosphonate and liposome scintigraphy with radiography. We also compare each investigation with respect to sensitivity in detecting clinically active disease.

\section{Patients and methods}

PAT IENTS

Thirteen patients with rheumatoid arthritis (RA), fufilling the American Rheumatism Association criteria for either definite or classical RA, ${ }^{9}$ were randomly selected at entry to a second line therapy trial and were studied by macroradiography of hands and wrists, ${ }^{49 \mathrm{~m}} \mathrm{Tc}$ methylene diphosphonate (MDP) bone scintigraphy ( ${ }^{99 \mathrm{~m}} \mathrm{Tc} \mathrm{MDP}$ ), and ${ }^{99 \mathrm{~m}} \mathrm{Tc}$ liposome scans. A further six patients had a clinical evaluation and liposome scan, of whom three also had an MDP bone scan.

Four patients with psoriatic arthritis were evaluated by clinical assessment, macroradiographs, and both liposome and MDP bone scan, while a further 
two patients had a clinical evaluation and liposome scan. One of these patients also had an MDP bone scan.

Four patients with osteoarthritis had clinical assessment, macroradiographs, and both bone scans and liposome scans.

CLINICAL ASSESSMENT

A modification of the Ritchie articular index ${ }^{10}$ was used to assess the degree of tenderness in the wrists $(0=$ non-tender, $1=$ tender, $2=$ tender and winced. $3=$ tender, winced, and withdrew).

J OINT IMAGING

Anteroposterior films of the hands and wrists were taken on non-screen film and separate macroradiographs of each hand produced. The radiographs were graded using a modification of the Larsen grading index as follows: grade $(0=$ normal; grade
$1=$ one or more of periarticular swelling, periarticular osteoporosis, joint space narrowing; grade $\overrightarrow{\overrightarrow{\mathrm{C}}}$ $2=$ erosion and joint space narrowing; grade $3=$ destructive changes where the original articular등 surfaces are lost.

Liposomes were prepared and radiolabelled with $\frac{\rho}{\widetilde{\Phi}}$ technetium- 99 as previously described. ${ }^{8}$ The patients' hands and wrists were imaged 20 hours afteres intravenous injection of $350 \mathrm{MBq}$ of ${ }^{99 \mathrm{~m}} \mathrm{Tc}$ labelled $\vec{\circ}$ liposomes. An image of both hands with palms towards the camera and containing 30000 countsw was acquired on an IGE 400A gammacamera ando the information stored on a link Dyanne dataô processor. A low energy parallel hole collimator was $\stackrel{\oplus}{\unlhd}$ used for all imaging of liposome distribution. के

${ }^{\left.{ }^{4}\right) \mathrm{m}}$ Tc MDP was obtained from Amersham Inter-op national. The patients' hands and wrists were similarly imaged approximately two hours after the intravenous injection. In this case, however, $300000_{c}^{\vec{C}}$
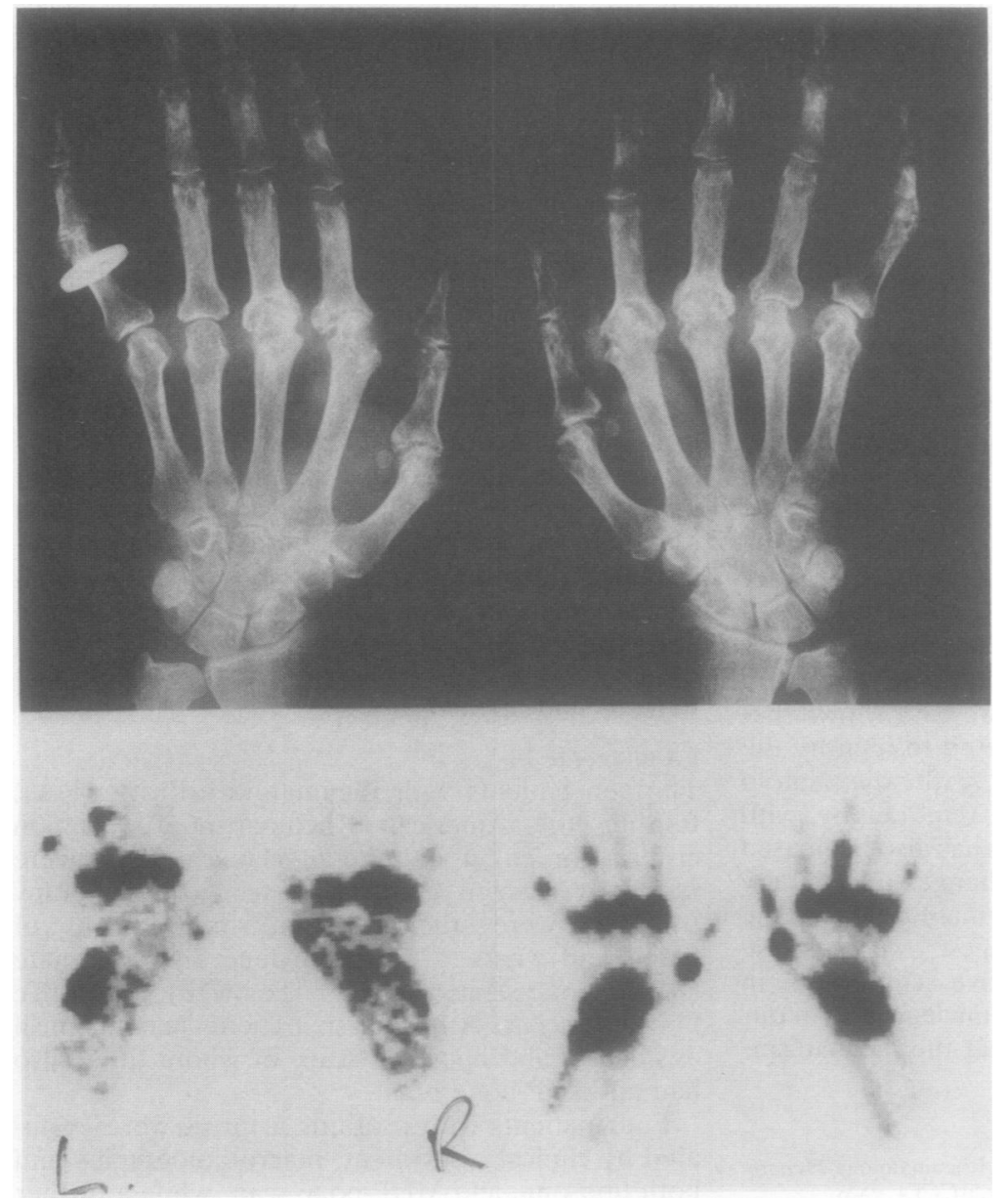

Fig. 1 Radiographs, liposome scan (bottom left), and bone scan (bottom right) of a patient with active seropositive $R A$. Note erosive changes at the metacarpophalangeal joints and increased activity in both scans. Note also the increased activity at the ulnar side of the left wrist on liposome scan but not on bone scan. Increased activity at the middle finger seen on bone scan is $\mathrm{N}$ not evident on the liposome scan. 
counts were acquired and a low energy high resolution parallel hole collimator was used.

Regions of interest were created using the Dyanne system for the whole hand, the carpus, the five metacarpophalangeal (MCP) joints as one region, and for the five hand digits. A background region was drawn between the carpus and the MCP joints. The counts in each region of interest were then randomised to unit area and the ratio for each normalised count to the background normalised count determined.

\section{STATISTICS}

Wilcoxon's sum of rank rests for unpaired data was used to compare scores between different groups.

\section{Results}

All clinical assessments, radiographic and scinti- graphic grading were done independently, and the observers were blind to the results of the other assessments. Fig. 1 shows the results of the three investigations in a patient with active seropositive erosive rheumatoid arthritis. The radiograph showed erosions in the wrists, MCP, and proximal interphalangeal (PIP) joints, which are the areas of increased uptake in both the liposome scans and the bone scan. The bone scan, however, showed diffuse uptake in the wrists, whereas the liposome scan showed more focal intake around the synovial proliferation over the ulnar head, particularly on the left side. The bone scan showed increased activity over the right third PIP and left first MCP, which was not evident on the liposome scan. In most patients with active disease, however, very similar patterns of activity were seen with both the bone scan and the liposome scans. In patients with inactive RA the liposome scans were normal even

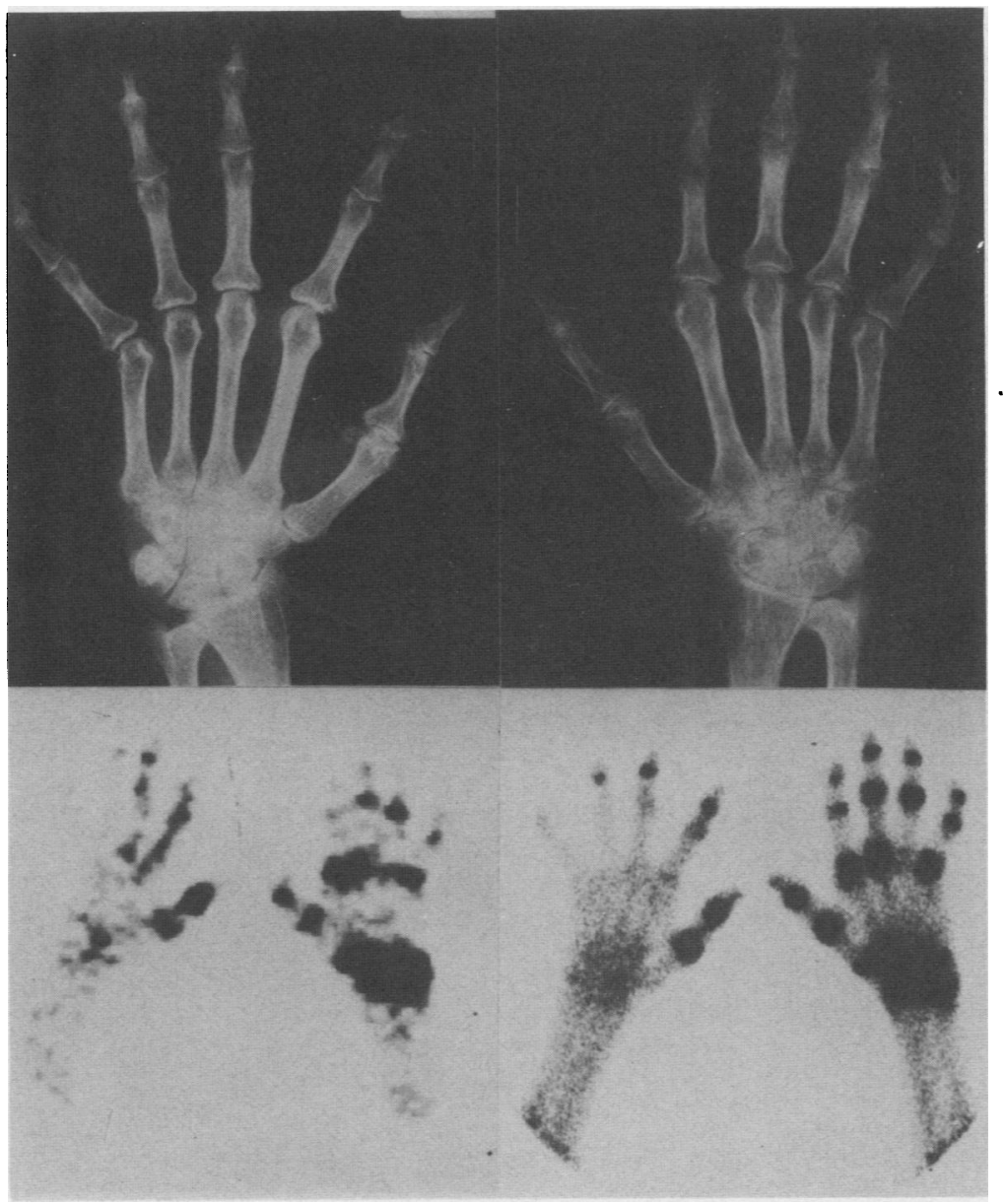

Fig. 2 Radiographs, liposome scan (bottom left), and bone scan (bottom right) of a patient with active psoriatic arthritis. Note loss of cartilage at wrists and erosions at metacarpophalangeal, proximal interphalangeal, and distal interphalangeal joints. Note also increased activity at these joints, especially the right hand on the bone scan. The liposome scan also shows increased activity, but there is more diffuse activity over the left index finger where clinically there was active tenosynovitis. 
though the bone scan showed increased uptake at the wrists and some MCP and PIP joints.

In psoriatic arthritis the radiographic changes were similar to those in RA, with periarticular osteoporosis and erosions in the small joints, including the distal interphalangeal (DIP) joints (Fig. 2). The bone scan again mirrored the radiological changes, and even though the liposome scan was positive, there were interesting differences between the scans. The liposome scan showed the tenosynovitis of the left index finger, which clinically had a sausage digit appearance. The diffuse uptake did not correspond with the more localised area of uptake over the DIP joint visible on the bone scan. In a patient with arthritis mutilans, however, the extensive osteolysis was evident on the radiographs and bone scan (Fig. 3), but in the absence of synovitis there was no uptake evident on the liposome scan of the small joints. The liposome scan failed to identify areas of inactive disease even though there was. extensive joint destruction.

Fig. 4 shows the three investigations in a patiento with osteoarthritis, who as well as having cystic듬 changes in the wrists had an acutely tender $\frac{\bar{c}}{\frac{1}{a}}$ Bouchard's node over the PIP joint of the third $\mathbb{Q}_{\square}^{\mathbb{Q}}$ finger on the right hand. A Heberden node over theg DIP joint of the same finger was also tender. Theses clinically painful and tender joints were identified? on bone scan and also by the liposome scan, though $\overrightarrow{\vec{\omega}}$ the degree of uptake appeared less than that observed in patients with RA or psoriatic arthritis. In non-inflammatory osteoarthritis Heberden $s_{A}^{\circ}$ nodes were present over the DIP joints, but these. joints were non-tender and associated with in $-p$ creased activity only on the bone scan.

We also compared the scintigraphic and radio-o graphic findings with the clinical assessment of joint inflammation. The patients with RA and highc

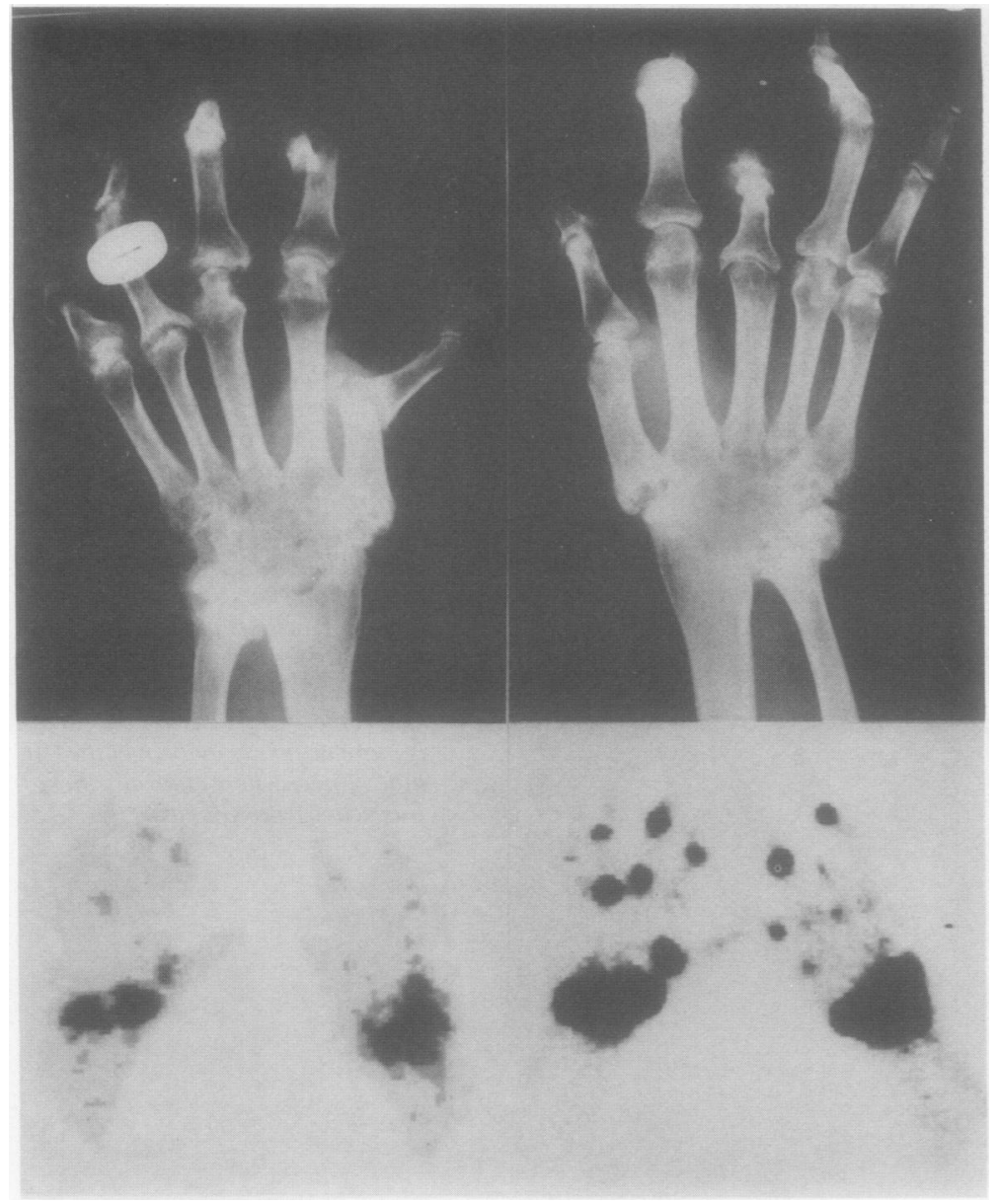

Fig. 3 Radiographs, liposome scan (bottom left), and bone scan (bottom right) of a patient with arthritis mutilans (PA). Extensive destructive changes of both wrists and fingers are seen. The bone scan shows increased activity, but very little activity is evident on the liposome scan, except over the wrists. 


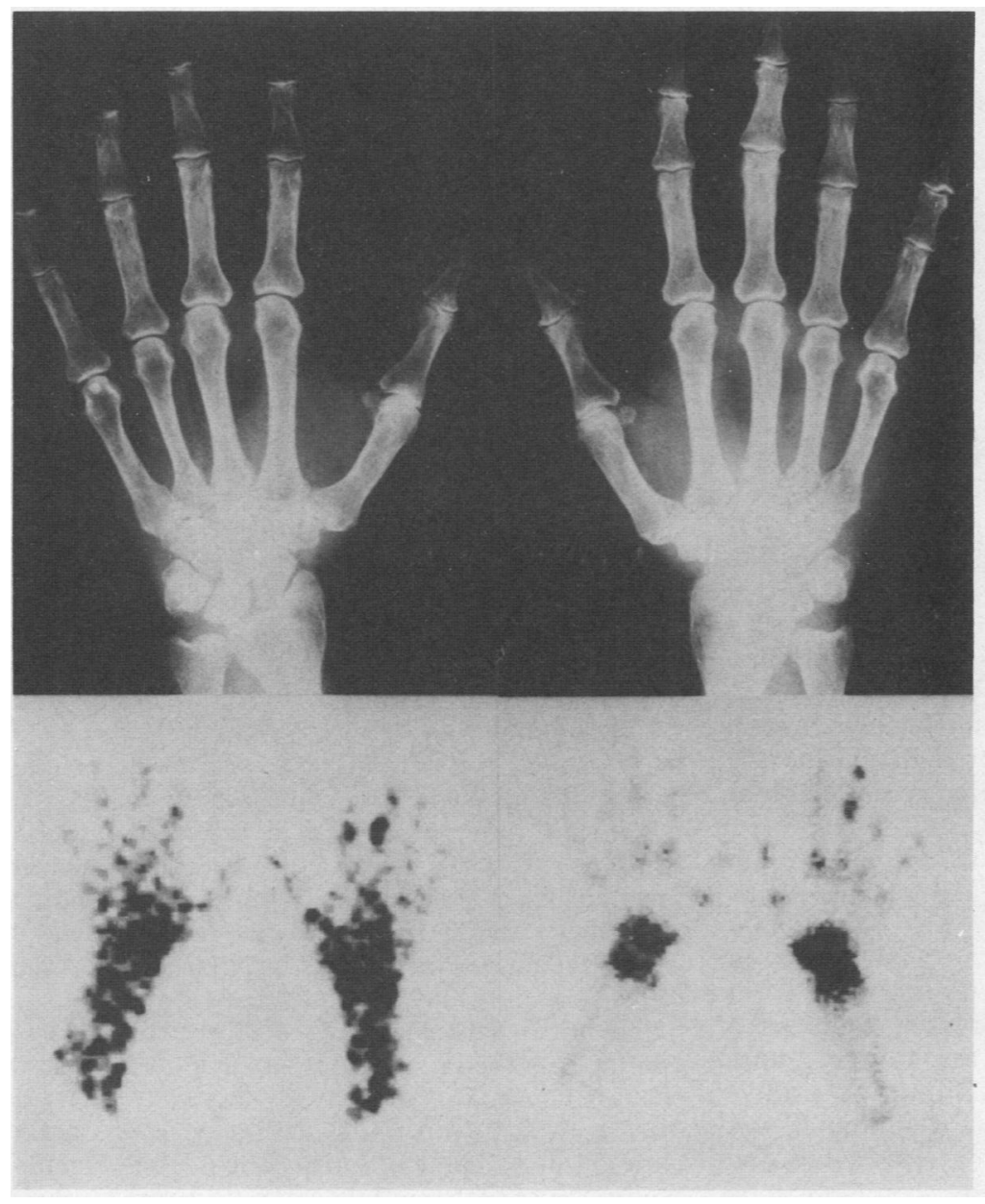

Fig. 4 Radiographs, liposome scan (bottom left), and bone scan (bottom right) of a patient with nodal osteoarthritis. Note the loss of cartilage at both wrists, erosion of the right middle finger distal interphalangeal joint and the increased activity on both scans. Bouchard's node at the right middle proximal interphalangeal joint shows little abnormality on the radiograph, but there is activity on both scans.
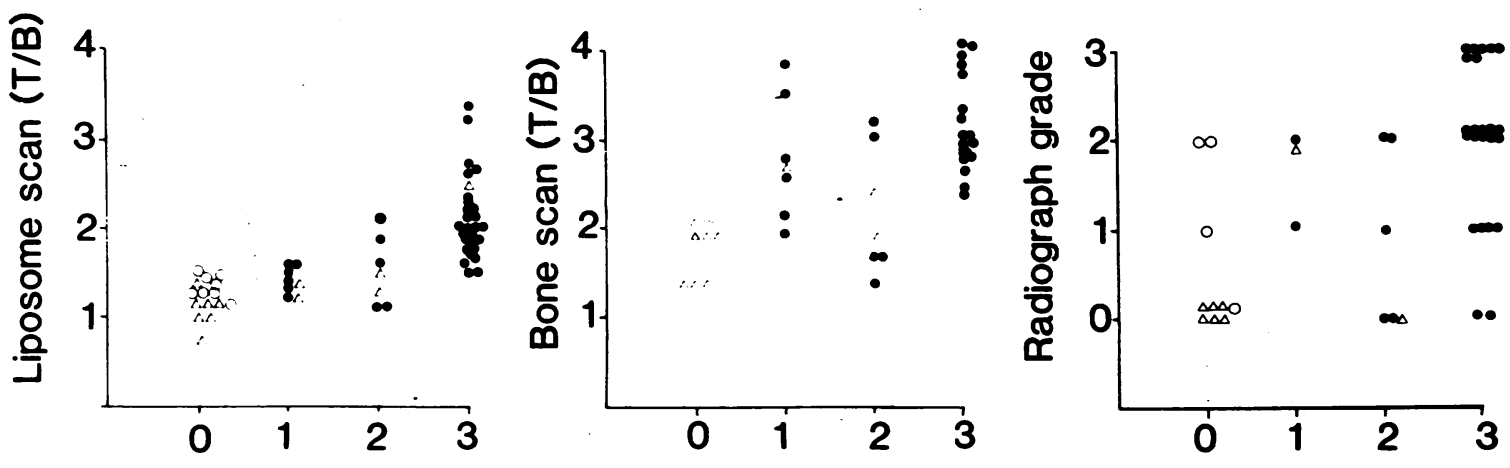

\section{Clinical index}

Fig. 5 Comparison of liposome scans, $M D P$ bones scans (target to background $(T / B)$ ratio), and radiographs (grade) with the clinical grading of joint tenderness in wrists of patients with rheumatoid arthritis $(O)$, psoriatic arthritis $(\triangle)$, and osteoarthritis (O). 
tenderness scores had higher target to background ratios (Fig. 5). The patients with osteoarthritis and psoriatic arthritis were scanned primarily to look at the DIP joints, and therefore the wrists scans served as controls for the patients with RA. The mean target to background (T/B) ratio for all patients judged clinically to be grade $3(2 \cdot 08)$ was significantly higher than those judged to be grade $0(1 \cdot 21)$ $(p<0 \cdot 01)$. The mean T/B ratios for the groups of patients with RA were $2.06,1.54$, and 1.49 for groups 3,2, and 1 respectively. When the groups of patients with RA were compared a significant difference was again seen between the mean T/B ratios for group 3 compared with group $1(\mathrm{p}<0 \cdot 01)$ and group $2(\mathrm{p}<0 \cdot 05)$, but the difference between group 1 and group 2 was not significant at the $5 \%$ level. All the patients with osteoarthritis who had liposome scans had non-tender joints and normal scans. Of the patients with psoriatic arthritis, seven joints were non-tender, and the scans were normal. Two wrists were grade 1 and two were grade 2 , and these had positive scans, while one wrist which was clinically grade 3 had a high T/B ratio $(2 \cdot 41)$. The numbers in these groups were not sufficient for adequate statistical comparisons.

The bone scans were more sensitive in discriminating between clinically active and inactive joints. The mean $\mathrm{T} / \mathrm{B}$ ratio for grade 0 was 1.81 and for grade 1 was $2 \cdot 76$. This difference was significant at the $1 \%$ level. The mean $\mathrm{T} / \mathrm{B}$ ratio for grade 3 was $3 \cdot 20$. In the patients with RA, however, there was a wide scatter of $\mathrm{T} / \mathrm{B}$ ratios among the different grades of joint tenderness; the mean $\mathrm{T} / \mathrm{B}$ ratio for grade 3 was 3.2 and for grade 1 was $2 \cdot 79$. This difference was not significant at the $5 \%$ level. There was also no significant difference between the scores obtained for grade 3 and those for grade $2(2 \cdot 20)$. The plain radiographs were the least able to discriminate between clinically tender and non-tender joints, with a wide scatter of radiological abnormality within each clinical grade group.

\section{Discussion}

These studies confirm our earlier findings that radiolabelled liposomes accumulate in inflamed joints. ${ }^{58}$ Although this was most clearly seen in patients with active rheumatoid and psoriatic arthritis, it also occurred occasionally in patients with inflammatory osteoarthritis. When comparisons were made, however, with the radiographs and the bone seeking MDP scans it was apparent that the bone scan was the most sensitive method of detecting clinically active disease. Previous studies have alluded to the increased sensitivity of bone scanning over radiography. ${ }^{11}$ In the quantitative assessment of joint tenderness in rheumatoid arthritis, however, the liposome scans were best able to discriminate $\overrightarrow{\overrightarrow{\vec{m}}}$ between different degrees of joint tenderness. This $\overrightarrow{0}$ finding is a little surprising given that joint tender- $\frac{\bar{\sigma}}{\sigma}$ ness may arise from the subcutaneous tissues, $\overline{\bar{D}}$ pericapsular, capsular, synovial, and bony structures, $\widehat{\mathbb{D}}$ with the contribution of each varying with different types of joint disease.

In inactive inflammatory polyarthropathies, $\vec{\circ}$ however, there are clear differences. It appears $\overrightarrow{\vec{\omega}}$ that once inflammation has initiated joint damage $\mathrm{S}$ the bone scan continues to reflect bone damage, $\frac{0}{2}$ whereas the liposome scan more accurately reflects the clinical picture of inactive disease. Weissberg. et al in their study of bone scanning in RA showed that in three patients with subjective improvemento after treatment there was no record of change in을 nuclear scanning. ${ }^{7}$ Similarly, Desauliers et al re-ported that in eight of 16 patients imaged before and $c$ during treatment, there was concordant progression of scan and clinical course. ${ }^{11}$ In the other eight the scans continued to show disease despite clinical $\infty$ improvement, or they became worse without any corresponding change of clinical status.

The few differences seen in active disease between the two scans do, however, suggest more $\overline{0}$ specific localisation of the liposomes to synovial\% tissue in inflamed joints and the tendon sheaths. $\mathbb{Q}$ These findings suggest, therefore, that the two scans $\overrightarrow{\overrightarrow{0}}$ reflect different disease processes. In active in- 3 flammatory polyarthropathies both mechanisms operate, but the bone scan is less able to reflect the? change in clinical state from active to inactive disease. Much work to date has centred on the? mechanism of uptake of the bone seeking agents. Studies have confirmed the role of increased blood flow ${ }^{12}$ and binding of the diphosphonate to the $\frac{0}{3}$ hydroxyapatite crystal, ${ }^{13}$ but there is also evidence that binding onto organic components of bone matrix, such as collagen, may be important. ${ }^{14}$ ?

We are currently investigating the mechanisms responsible for producing positive liposome scans in $N$ patients with RA. They are not produced by liposome accumulation in synovial fluid as the scans do not change after the aspiration of large volumes $\omega$ of synovial fluid from the joint. Scanning at 20 hours does not provide information on blood poolinge within the inflamed synovium because our earlier $\cong$ studies have clearly shown that between four and $20^{-}$ hours, when blood radioactivity falls, there is a ${ }^{\circ}$ significant increase in the accumulation of tech- $\overrightarrow{\mathbb{D}}$ netium within the joint. More direct evidence of the $\frac{?}{\mathbb{D}}$ synovial localisation of technetium has been $\cong$ obtained from a patient with RA who received ${ }^{99 \mathrm{~m}}$ Tc labelled liposomes 20 hours before under- 8 going surgery for a knee replacement. The synovialo 
tissue contained significantly more radioactivity than did muscle or synovial fluid and it remained associated with cells that were released from the synovium after enzymatic digestion. Although more work is required in this area, these observations would certainly be consistent with the idea that a positive scan reflects phagocytosis of radiolabelled liposomes by macrophages present in synovial tissue.

The abnormality is not confined only to rheumatoid arthritis but is also seen in psoriatic arthropathy, where an inflammatory synovitis with mononuclear cell infiltration similar to RA is seen. It is interesting to note that positive liposome scans are also seen in the inflammatory subgroup of osteoarthritis. Recent work on bone scanning in generalised nodal osteoarthritis has commented on the marked dissimilarity in severity of involvement on $x$ ray compared with MDP scan, ${ }^{15}$ suggesting that the scan reflects a different process from the anatomical change seen on radiographs. We suggest that liposome scanning may be able to help in differentiating between these subgroups. In our study only those patients with clinical evidence of inflammation had positive liposome scans, while in the non-inflammatory subgroup of osteoarthritis, even though the bone scan was positive, there was no increased activity on liposome scan.

Despite the lack of specificity associated with the use of radiolabelled liposomes in inflammatory joint disease our studies suggest that, even allowing for the limitations of using a single parameter to assess joint inflammation, it may be a sensitive indicator of changes in the clinical state. Determination of the factors contributing to a positive scan or whether this technique offers any advantages in monitoring disease progression must await further studies.

\section{References}

1 Gregoriadis G. The carrier potential of liposomes in biology and medicine. $N$ Engl J Med 1967; 295: 704-10. 756-72.
2 Richardson V J, Jeysingh K. Jewkes R F. Ryman B E. Tathersall M H N. Properties of ${ }^{44 \mathrm{~m}}$ technetium-labelled liposomes in normal and tumour bearing rats. Biochem Soc Trans 1977: 5: $290-3$.

3 Richardson V J, Ryman B E, Jewkes R F, et al. Tissue distribution and tumour localisation of ${ }^{99} \mathrm{~m}$ technetium labelled liposomes in cancer patients. Br J Cancer 1979; 40: 35-43.

4 Morgan J R, Williams L A. Howard C B. Technetium labelled liposome imaging for deep seated infection. Br J Radiol 1985: 58: $35-9$.

5 Williams B D. O’Sullivan M M. Saggu G S. Williams K E. Williams L A. Morgan J R. Imaging in rheumatoid arthritis using liposomes labelled with technetium. $\mathrm{Br}$ Med J 1986; 293: 1143-4.

6 Bekerman C. Genant H K. Hoffer P B. Zokin F. Ginsberg M. Radionuclide imaging of the bones and joints of the hand. Radiology 1976: 118: 653-9.

7 Weissberg D. Resnick D. Taylor A. Becker M, Alazraki N. RA and its variants: analysis of scintiphotographic, radiographic and clinical examination. $A J R$ 1978; 131: 665-73.

8 Williams B D. O'Sullivan M M. Saggu G S, Williams K E. Williams L A, Morgan J R. Synovial accumulation of technetium labelled liposomes in rheumatoid arthritis. Ann Rheum Dis 1987; 46: 314-8.

9 Ropes M W, Bennett G A, Cobb S, Jacox R T, Jessar R A. 1958 revision of diagnostic criteria for rheumatoid arthritis. Arthritis Rheum 1959; 2: 16-20.

10 Ritchic D M. Boyle J A. McInnes J M. et al. Clinical studies with an articular index for the assessment of joint tenderness in patients with rheumatoid arthritis. $Q J$ Med 1968; 37: 393-406.

11 Desauliers M. Fuks A. Jawkins Y. Lacourchicre Y. Rosenthall L. Radiotechnetium polyphosphate joint imaging. $J$ Nucl Med 1974; 15: 417-23.

12 Genant H K. Bautovich G J. Singh M. Bone secking radionuclides: an in-vivo study of factors affecting skeletal uptake. Radiology 1974; 113: 373-84.

13 Jung A, Bisaz S. Fleisch $H$. The binding of pyrophosphate and two diphosphonates by hydroxyapatitc crystals. Calcified Tissue Research 1973; 11: 269-80.

14 Rosenthall L. Kaye M. ${ }^{* / n}$ Technetium pyrophosphate kinctics and imaging in metabolic bonc discasc. J Nucl Med 1975: 16: 33-9.

15 Hutton C W. Higgs E R. Jackson P C. Watt I. Dicppc P A. ${ }^{9 y_{m} \mathrm{~T}} \mathrm{TcHMDP}$ bone scanning in generalised nodal ostcoarthritis. I. Comparison of the standard radiograph and four hour bone scan image of the hand. Ann Rheum Dis 1986; 45: 617-21. 